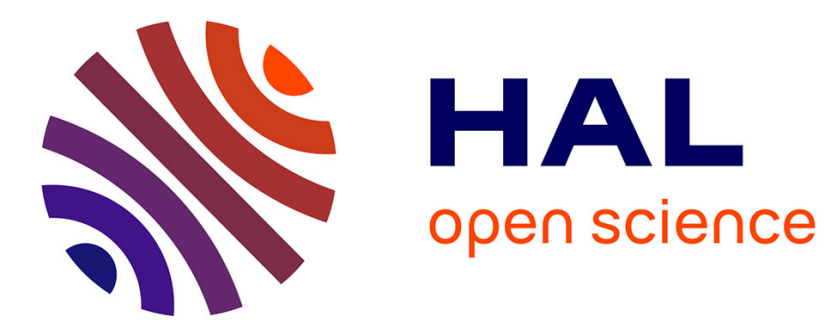

\title{
Présence des royautés Sud-Est asiatiques
}

\author{
Bernard Formoso, Gregory Mikaelian, Marie-Sybille de Vienne
}

\section{To cite this version:}

Bernard Formoso, Gregory Mikaelian, Marie-Sybille de Vienne. Présence des royautés Sud-Est asiatiques. Jérémy Jammes et François Robinne. L'Asie du Sud-Est 2014, bilan, enjeux et perspectives, Indes savantes/IRASEC, pp.99-121, 2014. hal-01422290

\section{HAL Id: hal-01422290 \\ https://hal.science/hal-01422290}

Submitted on 24 Dec 2016

HAL is a multi-disciplinary open access archive for the deposit and dissemination of scientific research documents, whether they are published or not. The documents may come from teaching and research institutions in France or abroad, or from public or private research centers.
L'archive ouverte pluridisciplinaire HAL, est destinée au dépôt et à la diffusion de documents scientifiques de niveau recherche, publiés ou non, émanant des établissements d'enseignement et de recherche français ou étrangers, des laboratoires publics ou privés. 


\section{Présence des royautés Sud-Est asiatiques}

\section{Réflexions sur la base d'un texte de Grégory Mikaelian et Marie-Sybille de Vienne, associant Bernard Formoso (Thaïlande)}

En passer par la notion de royauté pour comprendre l'évolution contemporaine des États d'Asie du Sud-Est permet d'appréhender un large éventail de situations. Si quatre seulement de ces États ont conservé un souverain (Thaïlande, Cambodge, Malaisie, Brunei), il est admis par nombre d'analystes que certains pays ayant formellement aboli la royauté connaissent de plus ou moins fortes rémanences d'un système de légitimation royal, alors même que leur gouvernement prétend rompre avec cette forme de gestion décentralisée des sociétés (cas du Myanmar, du Viêt Nam et du Laos) ${ }^{1}$. La principale

\footnotetext{
${ }^{1}$ Par exemple, Roger Kershaw distingue à l'échelle de l'Asie du Sud-Est et dans le cadre d'une grille d'analyse biaisée assimilant la monarchie à la royauté: une «ruling Monarchy " (Brunei), trois " monarchies constitutionnelles» (Cambodge, Malaisie, Thaïlande), et des pays pour lesquels l'héritage "monarchique» est pertinent (Laos, Indonésie), v. R. Kershaw, Monarchy in Southeast Asia. The Faces of Tradition in Transition, Paris, London \& New York, Routledge, 2001, 268 p. Pour le cas de la Birmanie, v. Jacques Népote, «La Birmanie post-coloniale (1942-1992). Histoire manquée d'une tentative de reconstruction culturelle ? », Péninsule n 24-25, 1992, p. 105-128 ; Marie-Sybille de Vienne, "La Birmanie entre modernité et légitimité, à la recherche d'un nouveau roi Cakravartin », in Les structures politiques traditionnelles à l'épreuve de la démocratie en Asie du Sud-Est, Péninsule n 48, 2004 (1), p. 139-158). Dorothy Guyot, « The Uses of Buddhism In Wartime Burma ", in International Conference on Asian History, 5th-10th Aug. 1968, Kuala Lumpur, Univ. of Malaya, Dept. of History, 1968, p. 67 ; Donald M. Seekins, The disorder in order, the Army-State in Burma since 1962, Bangkok, White Lotus Press, 2002, p. 50 ; Pour le Laos, v. Grant Evans, "Royal Family Commemorative Rituals Today », in The Last Century of Lao Royalty, Chiang Mai, Silkworm Books, p. 419-420. Pour le cas du Viêt Nam, v. Paul Mus, Viêt-nam, sociologie d'une guerre, Paris, Editions du Seuil, Collections Esprit «frontière ouverte ", 1952, 378 p. ; idem, Ho Chi Minh, Le Vietnam, L'Asie, Paris, Le Seuil, 1971, 251 p. ;
} 
caractéristique des royautés de la zone était en effet leur aspect segmentaire ${ }^{2}$, trait commun à nombre de royautés anciennes ${ }^{3}$. Ailleurs encore, ce sont nombre de pratiques politiques qui illustrent une grammaire du pouvoir procédant à maints égards du fait royal, à tout le moins conforme au souvenir qu'en ont gardé une partie des élites (Indonésie, Timor, Philippines) ${ }^{4}$. Si bien qu'un tel angle d'approche permet d'aborder en profondeur les traditions politiques de la zone, au sens de l'actualisation, dans le présent, des pratiques héritées du passé ainsi que leur cortège de symboles associés.

À travers les survivances et les rémanences dont les royautés font montre, y compris là où elles ont formellement disparu, se pose la question des modalités de leur adaptation dans une économie de marché mondialisée d'inspiration libérale. D'origine européenne ${ }^{5}$, cette modernité vient en effet se greffer sur une modernité sud-est asiatique qui la précédait et qui continue de façonner les sociétés contemporaines. On a pu diversement la qualifier de " première modernité $»^{6}$, de « temps des réseaux asiatiques $»^{7}$ ou $\mathrm{d}^{\prime}$ «âge du commerce $»^{8}$. Elle suscita parmi

Nguyên Duc Nhuân, "Du Viêt-nam communiste au Viêt-nam des "cent maisons" », Le Monde Diplomatique, septembre 1992, p. 20-21.

${ }^{2}$ Stanley Jeyaraja Tambiah, « The Galactic Polity: The Structure of Traditional Kingdoms in Southeast Asia ", Annals of the New-York Academy of Sciences, vol. 293, 1977, p. 69-87 ; Nguyên Thê Anh, "La féodalité en Asie du Sud-Est », in Eric Bournazel et Jean-Pierre Poly (dir.), Les féodalités, Paris, Presses Universitaires de France, 1998, p. 683-714.

${ }^{3}$ Dans sa généralité, l'État segmentaire se définit comme « une configuration politique dont la souveraineté territoriale est reconnue mais limitée, l'autorité étant de plus en plus diffuse à mesure qu'elle s'éloigne du centre, le gouvernement central coexistant avec des foyers de pouvoir relativement autonomes, le monopole de l'exercice légitime de la force par l'État restant contesté, et les relations sociales reposant surtout sur des liens de personne à personne fondés sur une économie généralisée du don », v. Nicolas Le Roux, "Courtisans et favoris : l'entourage du prince et les mécanismes du pouvoir dans la France des guerres de religion ", Histoire, économie et société, 1998, vol. 17 (3), p. 379.

4 Denys Lombard, Le carrefour javanais. Essai d'histoire globale, t. I, Les limites de l'occidentalisation, Paris, EHESS, 1990, 267 p. ; M.-S. de Vienne, "Sulu-Jolo : l'histoire sans fin d'un sultanat qui refuse de mourir », Outre-Terre, n², mai 2002, p. 293-312); Douglas Kammen, «Queens of Timor », Archipel n 84, 2012, p. 149-173.

${ }_{5}^{5}$ Jean-Claude Michéa, La double pensée. Retour sur la question libérale, Paris, Flammarion, 2008 , p. $197 ; 199-200 ; 203-204$.

${ }^{6}$ Sanjay Subrahmanyam, « Connected Histories: Notes towards a Reconfiguration of Early Modern Eurasia ", [in] Beyond Binary Histories. Re-imagining Eurasia to c. 1830, Lieberman, Victor (dir.), Ann Arbor, The University of Michigan Press, 1999, p. 289-316.

${ }^{7}$ D. Lombard, op. cit., t. II, Les réseaux asiatiques, Paris, EHESS, 1990, 423 p. 
les royautés de la région une refonte des institutions comme de l'exercice du pouvoir, à la fois pour s'adapter aux nouvelles conditions mercantiles induites par l'accélération des échanges maritimes, mais aussi pour en pallier les effets socialement dissolvants (le déplacement des sites de pouvoir et l'avènement des bourgeoisies, pour autant qu'on puisse employer ce terme lapidaire). Dans ce cadre, l'adoption de nouveaux programmes religieux (bouddhisme theravāda, islam, christianisme), qui accompagnaient autant qu'ils nourrissaient ces bouleversements, entraîna une refonte des politiques de la représentation royale. Pour certaines des entités en cause (Birmanie, Siam, Viêt Nam en Péninsule ; Malacca, Java [Mataram] et Aceh en Insulinde), le processus s'est accompagné d'une expansion politique aux dépens des voisines ${ }^{9}$ et sur laquelle l'État colonial est venu s'abouter. Si l'on souhaite comprendre les régimes politiques contemporains, il faut donc commencer par rappeler d'où ils viennent.

L'exemple cambodgien et le cas de Brunei sont particulièrement révélateurs en ce qu'ils procèdent de généalogies remontant aux débuts de l'Histoire. Le cas thaïlandais intéresse à l'inverse pour sa relative jeunesse.

\section{1 - La royauté cambodgienne}

Le royaume du Cambodge offre le cas d'un régime politique qui puise ses fondements idéologiques, dans le souvenir mythographique de la première entité indianisée de la zone, le Funan (c. 0-VIe siècles). C'est en effet durant cette "première Angkor ${ }^{10}$ que s'est formalisé le mythe de fondation dynastique, lequel n'a cessé par la suite d'être reconfiguré

\footnotetext{
8 Anthony Reid, Southeast Asia in the Age of Commerce 1450-1680, New Haven and London, Yale University Press, 1988-1993, 2 vol., 275 et 390 p.

9 Victor Lieberman, Strange Parallels. Southeast Asia in Global Context, c. 800-1830, Cambridge, Cambridge University Press, 2 vol. 2003-2009 484 et 947 p. ; A., Reid, op. cit.

${ }^{10}$ Eric, Bourdonneau, «Réhabiliter le Funan. Óc Eo ou la première Angkor », BEFEO, t. 94, 2007 [2010], p. 111-158.
} 
par les rois khmers jusqu'au XVII e siècle ${ }^{11}$, moment de refondation clef du pouvoir après le sac de Lañvaek par les Siamois en 159412. Mythe dont procède aujourd'hui encore le fonctionnement de la royauté cambodgienne. Celui-ci vise à magnifier l'étranger en l'intégrant au creuset autochtone par une alliance fécondante. Cette manière de fonder (ou refonder) le pouvoir par le truchement d'une exaltation de l'altérité figure comme le trait liminaire d'une royauté qui ne cessa, au cours de l'histoire, de restructurer ses assises en empruntant à des cultures étrangères pour mieux perpétuer son être. Le problème est que les cultures choisies pour concourir au titre d'alter ego référent ont été de plus en plus éloignées de l'environnement cambodgien et que cette pratique héritée de recourir à un vocabulaire étranger pour construire une vision du monde qui fasse sens hic et nunc en est venue progressivement à heurter certaines pratiques sociales, comme les règles d'alliance et de filiation. Au point que ladite vision a tendu à ne plus être aussi bien partagée qu'elle avait pu l'être durant les temps de l'indianisation. D'un apport en provenance d'un sous-continent indien protohistorique, sans doute en partie mâtiné de culture austroasiatique, à la taïsation progressive du royaume khmer, à la pénétration d'éléments culturels de Chine du Sud associés aux valeurs mercantiles du Nanyang, et jusqu'à l'occidentalisation libérale, c'est à un écart toujours plus grand entre pratiques importées et pratiques locales qu'ont dû faire face les élites cambodgiennes.

L'époque moderne aura ainsi vu s'épanouir une manière de seconde indianisation par le biais de la bouddhisation theravādine du royaume qui, à bien des égards, a correspondu à une siamisation des cultes et des mœurs curiales - d'autant plus subtile que la royauté siamoise s'est historiquement construite sur un palimpseste de pratiques culturelles môn, khmères et péariques ${ }^{13}$ progressivement taïsées du XIVe

\footnotetext{
${ }^{11} \mathrm{~J}$. Népote, «Mythes de fondation et fonctionnement de l'ordre social dans la basse vallée du Mékong accompagnés de considérations sur l’indianisation ", Péninsule n³8, 1999 (1), p. 35-67.

$12 \mathrm{G}$. Mikaelian, La royauté d'Oudong. Réformes institutionnelles et crise du pouvoir dans le royaume khmer du XVII siècle, Paris, Presses Universitaires de la Sorbonne (PUPS), 2009, $374 \mathrm{p}$.

${ }^{13}$ Le péarique (du groupe Pear) est une branche du môn-khmer.
} 
au XIXe siècle ${ }^{14}$. C'est essentiellement à cette époque dite postangkorienne ou moyenne qu'il faut attribuer l'essentiel du legs traditionnel $\mathrm{du}$ fonctionnement politique actuel. Le peu que l'on en connaît montre qu'elle irrigue plusieurs constantes de la vie politique cambodgienne $^{15}$, et notamment la politique extérieure qui reproduit typiquement, depuis l'indépendance, le jeu «galactique » décrit par l'anthropologue Stanley Tambiah pour la royauté ${ }^{16}$. Quand la divinité tutélaire du royaume (le neak ta khleaing muong17 revêt également des atours siamois (le "centre de la Cité ", du siamois klang muong18), le monachisme s'y organise pareillement en deux sectes bouddhiques suivant en cela un modèle tardif importé depuis Bangkok sous le règne d'Ang Duong (1847-1860): l'ordre royal dit Dhammayuth et l'ordre populaire dit Mahānikay.

La dualité de l'exercice du pouvoir - qui procède d'une généalogie longue dans la mesure où elle puise probablement au système de filiation dynastique angkorien ${ }^{19}$ - se présente, elle aussi, à l'époque moderne, comme une innovation bouddhique. Elle s'exprime par exemple à travers la récurrence de l'opposition entre un roi de justice (dhammarāja) de filiation reconnue, et un homme pourvu de mérites ( $p h u$ mi bun en siamois, qnak mān puny en khmer) sorti du rang par ses hauts faits, catégorie à laquelle est réputé appartenir l'actuel Premier ministre $\mathrm{du}$ Cambodge, Hun Sen. Ce dernier n'a eu d'ailleurs de cesse d'encourager cette identification sur les avis d'un conseiller républicain qui avait lui-même établi des recherches (fort peu) historiques sur le "roi des partisans" (Sdech Kan): un personnage légendaire des chroniques royales qui figure comme l'archétype du pourvu de mérites

\footnotetext{
14 Michael Vickery, "A new Tamnan about Ayudhya », Journal of the Siam Society, $\mathrm{n}^{\circ}$ 67, 1979, p. 123-186.

15 Serge Thion, "Quelques constantes de la vie politique cambodgienne ", Affaires cambodgiennes, 1979-1989, Paris, L'Harmattan, Asie-Débat - 5, 1989, p. 224-247.

16 Nasir Abdoul-Carime, "Mise en perspective de la diplomatie sihanoukienne: une logique doublement péninsulaire et de temps long », Péninsule, n 36, 1998 (1), p. 175-191.

${ }^{17}$ qnak tā ghlāmni mìoen.

18 klārimìoeri.

${ }^{19}$ M. Vickery, «The Reign of Sūryavarman I and Royal Factionalism at Angkor », Journal of Southeast Asian Studies, vol. XVI, n² 2, sept. 1985, p. 226-244 ; É. Bourdonneau, Indianisation et formation de l'État en Asie du Sud-Est : retour sur trente ans d'historiographie. Matériaux pour l'étude du Cambodge ancien, Paris, Université de Paris I, 2005, p. 474.
} 
renversant la tyrannie du roi en titre, le tout dans un décor du premier quart du XVIesiècle. Après avoir usurpé le statut de personnage historique rebelle à l'autoritarisme des rois sous la République de Lon Nol (1970-1975), le Sdech Kan poursuit son rôle de légitimation des puissants confrontés à l'épineuse question de la légitimité «naturelle » du Trône ${ }^{20}$. La dualité se traduit encore au sein même de la famille royale à travers la répartition récurrente du pouvoir entre un roi en titre et un roi ayant abdiqué, chacun se voyant attribuer un palais distinct. Ainsi, du roi Suramarith (r. 1955-1960), père de Sihanouk et résidant à Chatomukh, le palais royal de Phnom Penh, tandis que son fils abdicataire s'installait à Chamkar Mon, au sud de la ville, sorte de palais civil où ce dernier occupa les fonctions de chef de l'État à partir du milieu des années 1950. Ressort encore de ce schéma la bipartition du pouvoir royal entre Sihanouk, remonté sur le trône en 1993 avant qu'il n'abdique à nouveau en 2004, et son fils, le roi Sihamoni, qui dut composer avec l'aura paternelle jusqu'au décès du roi-père intervenu en octobre 2012. Il n'est pas jusqu'à la gestion des conflits fonciers qui ne relève d'un héritage de l'époque moderne. Le droit coutumier précolonial toujours en vigueur dans les campagnes reconnaît sans ambiguité l'existence de la propriété foncière, bien qu'elle ne s'exprime pas dans les termes de notre droit romain-germanique. Est propriétaire tout paysan qui cultive sa terre, à charge pour lui ne pas la laisser tomber en déshérence plus de trois ans, auquel cas il la perd. Sur cette base, les traités de sacre du XVII siècle (toujours en vigueur) stipulent que le roi dispose d'un droit d'allocation des terres sur tout le royaume (et non pas, $c^{\prime}$ est l'erreur des analystes coloniaux reprise en chœur par les spécialistes actuels du foncier, un droit de propriété éminente sur ces terres). Ce droit d'allocation se comprend, dans les termes de l'anthropologie juridique, comme une fictio legis, c'est-à-dire une disposition dont la fonction est de magnifier la souveraineté du roi. Est souverain celui à qui l'on reconnaît le droit d'allouer les terres du royaume (le privilège de dire à qui appartient telle parcelle, mais pas celui de l'exploiter à son profit), de même qu'il a le droit d'allouer les charges du royaume (mais pas de les cumuler sur sa personne). Concrètement, le roi usait

20 Astrid Norén-Nilsson, «Performance as (re)incarnation: The Sdech Kân narrative ", Journal of Southeast Asian Studies, vol. 44 (1), 2013, p. 4-23. 
ponctuellement de ce droit pour délocaliser un village afin d'y construire une route, ou pour en détruire un autre en punition d'une rébellion. Sur cette base, le Protectorat (1863-1953) est venu s'insérer dans le dispositif légal cambodgien en préemptant la souveraineté royale au profit du Conseil des ministres placé sous la coupe du Résident supérieur, avec cette conséquence qu'il s'est approprié ce droit d'allocation. La concession était née. L'État postcolonial continua d'agir sur ce mode, mais sans grands dommages pour la paysannerie car les concessions n'étaient pas légion. Avec la réouverture du Cambodge au tournant des années 1990, le pays s'est en revanche mué en "royaume concessionnaire ${ }^{21}$, les grands ministres monnayant à des fins privées l'allocation des terres au profit principalement d'investisseurs chinois d'outre-mer; avec les conséquences dramatiques que l'on sait pour les droits coutumiers des paysans, sommés d'arborer un titre de propriété sous peine d'être expropriés. En clair, le gouvernement a retourné la traditionnelle clause de souveraineté contre le droit coutumier, à l'aide du droit romano-germanique importé à l'époque coloniale, à ceci près que dans cette opération juridique son allié objectif aura été la Banque mondiale, qui en bon agent du libéralisme a financé une réforme foncière généralisant les titres de propriété, aux dépens de la petite paysannerie.

Si l'on invoque souvent le legs colonial pour éclairer la situation politique actuelle, ce n'est toutefois pas toujours à bon escient dans la mesure où les acteurs du changement sont rarement considérés en tant que tels. Après avoir fait l'indien, puis joué au siamois (aux XVIIe et XIXe siècles notamment), une partie de l'aristocratie khmère avait fait le choix de reproduire certaines manières de penser, de dire et de faire européennes. Elle s'associa pour cela à des sino-khmers ou des Khmers de Cochinchine au service du Protectorat, groupes traditionnellement placés en situation d'interface avec la modernitée ${ }^{22}$. Les aristocraties conservatrices ont ainsi été progressivement écartées du pouvoir (à

\footnotetext{
${ }^{21}$ J.-F. Bayart, R. Bertrand, B. Hibou, R. Marchal et F. Mengin, Le royaume concessionnaire. Libéralisation économique et violence politique au Cambodge, Paris, FASOPO, 2004, 242 p.

${ }^{22}$ J. Népote, "Les nouveaux sino-khmers acculturés : un milieu social perturbateur ? ", Péninsule $\mathrm{n}^{\circ}$ 30, 1995 (1), p. 133-154.
} 
commencer par les grands princes et la noblesse de robe) au profit de «jeunes Turcs », souvent d'anciens interprètes. Ces nouvelles élites, qui ont fini par remplacer les anciennes, ont eu toutefois une attitude ambivalente à l'endroit de la royauté. D'un côté, elles ont su jouer la carte de la famille royale pour s'introduire dans la place, en soutenant tel prince contre tel autre (Sisowath puis Monivong contre les fils Norodom ; Youthevong puis Sirik Matak contre Sihanouk, etc.). Mais, adoptant les idées progressistes européennes, elles ont dans le même temps œuvré de plus en plus ouvertement contre la royauté (des Issaraks aux Khmers rouges en passant par le Parti Démocrate et les Républicains) s'abritant derrière un nationalisme de façade qui reprenait à bon compte les symboles de l'autorité palatiale (ex. de l'hebdomadaire Nagara Vatta qui récupéra dans les années 1930 le thème d'Angkor Vat, pilier de la symbolique royale) tout en s'adossant aux vieilles forces centrifuges (gens du Nord et de l'Ouest, pro-siamois, contre les gens du Sud et de l'Est, pro-vietnamiens). À mesure de ce qu'elles s'acculturaient et se constituaient en bourgeoisie prédatrice exploitant les campagnes, elles ont concurrencé le discours traditionnel de la royauté sur la protection du peuple ( $c f$. l'idéologie du Sangkum Reastr Niyum, 19551970) par un discours emphatique sur la protection du territoire. Celui-ci était $\mathrm{mu}$ par une colonne vertébrale xénophobe aux vertus compensatoires: hurler à l'avalement des terres cambodgiennes et magnifier la khméritude à proportion de ce que l'on est coupé de la culture khmère (ainsi du «néo-khmérisme » du sino-khmer Lon Nol).

Après la rupture qu'a représentée la guerre civile en partie provoquée par les sanglantes interventions états-uniennes (1967-1989), on a vu émerger une nouvelle sociologie politique, avec la mise à l'écart des gens de l'Ouest (une forte composante des Khmers rouges) au profit $\mathrm{d}^{\prime}$ acteurs issus surtout du Sud-Est et de l'Est (Hun Sen et les cliques de Kompong Cham; Chea Sim et celles de Prey Veng ou de Svay Rieng), plus proches des réseaux sino-vietnamiens, et pour la plupart issus de familles qui étaient novices aux commandes de l'État. L'idéologie adoptée fut alors celle du rattrapage socio-économique consécutif au désastre des Khmers rouges, rattrapage que devait servir un mouvement de «reconstruction» du pays. Derrière de réels besoins et d'authentiques bonnes volontés, cette phraséologie a surtout servi de cache-misère à un affairisme débridé en appelant à toutes les maffias et 
tous les réseaux mercantiles de Chine d'Outre-mer puis d'Asie Orientale (Chine populaire, Corée du Sud). Ainsi parvenus aux affaires, ces clans ont reconstitué un système d'alliance classique autour des principaux hiérarques du régime qui se sont partagés les ressources du pays. Mais la lutte qui les a rapidement divisés (Hun Sen versus Chea Sim), ainsi que la pression internationale pour réintroduire les autres factions cambodgiennes dans la course lors du processus électoral de 1993 (Funcinpec, FNLPK, Khmers rouges) eurent pour effet de replacer la royauté personnifiée par Sihanouk en position d'arbitre. En plus d'être un faire-valoir légitimant aux yeux de la communauté internationale, la Couronne est alors redevenue le symbole d'une unité nationale que Hun Sen et son clan ne pouvaient incarner. D'autant qu'après des décennies de destruction, une véritable aspiration à la paix se faisait sentir. Celle-ci étant culturellement un attribut $\mathrm{du}$ bon gouvernement des rois bouddhiques, c'est quasi naturellement que Sihanouk a pu restaurer le Trône en position de légitimer l'ordre rétabli, tout en se posant comme observateur moral de l'exercice du pouvoir.

Avec cet inconvénient pour lui qu'il ne pouvait plus y participer qu'à la marge. La restauration de 1993 a, de la sorte, institué une dualité de valeurs entre le Palais et le gouvernement, sur fond d'inversion : au souci du peuple porté par un Palais demeurant à l'écart de la vie politique s'opposent les pratiques prédatrices des bourgeoisies affairistes qui composent le gouvernement et la haute administration. Mais alors que l'imitation constructive était l'apanage des aristocraties d'hier, les bourgeoisies d'aujourd'hui ânonnent le jargon des "sociétés civiles » anglo-saxonnes et singent les entrepreneurs chinois d'outre-mer, élargissant d'autant la fracture entre le peuple et les élites. Dans ces conditions, quelle aura été la stratégie du vieux roi pour les années à venir lorsqu'il renonça au trône en 2004? Abdiquer au profit de Sihamoni aux dépens d'autres fils plus remuants assurerait de cantonner la fonction royale dans un rôle de neutralité politique, ce qui en garantirait la pérennité dans un environnement où l'essentiel des clientèles au pouvoir ont porté allégeance au Premier ministre.

Aujourd'hui, le faible pouvoir du nouveau roi tient effectivement à ce qu'il ne peut bénéficier des réseaux de clientèles de son père, produits $\mathrm{d}^{\prime}$ un long règne. Ce d'autant moins qu'il n'est pas marié : la force des souverains tenait aussi à leur capacité de capitaliser les femmes, 
emblèmes des différents clans du royaume et qui sont comme des clefs pour actionner - ou neutraliser - les forces régionales centrifuges. Ayant pour l'heure proscrit l'idée d'un mariage, il se condamne dès lors à n'avoir qu'une influence restreinte sur les affaires du pays. Au reste, la Couronne khmère est loin de disposer ne serait-ce que du dixième des biens de la Couronne thaïlandaise, premier propriétaire foncier et deuxième actionnaire du royaume de Thaïlande. Le palais dépend donc largement du Premier ministre pour ce qui est de son budget de fonctionnement. Derrière la réelle émotion suscitée par la mort du roipère en octobre 2012, un symbole de transcendance dans une mer de matérialisme débridée, il faut bien voir que plus grand monde ne connaît les usages, que les princes sont marginalisés de la scène politique, que leur situation financière est souvent précaire et, qu'au vrai, la royauté cambodgienne fait pâle figure. Mais c'est à ce prix qu'elle coexiste avec la puissance d'un Hun Sen, lequel a bien compris le parti qu'il en pouvait tirer.

Reste qu'au Cambodge sans doute plus qu'ailleurs, quand le peuple cesse d'aimer, il cesse d'obéir. Les contradictions croissantes du système de corruption que dirige le Premier ministre, ainsi que le factionnalisme atavique du personnel politique cambodgien sont lourds de troubles à venir, qui redonneront peut-être au Palais une partie du pouvoir perdu dans la guerre civile.

\section{2 - La royauté en Thaïlande}

La royauté du Siam, puis de Thaïlande, a imprimé sa marque sans discontinuité depuis plus de sept siècles sur la culture politique d'un pays qui est le seul dans la région à ne pas avoir été colonisé. À ce titre, la royauté est devenue, en osmose avec le bouddhisme theravāda qui légitime son autorité et sa fonction d'ordonnancement, l'une des deux institutions consubstantielles aux idées de souveraineté collective et d'identité nationales. En témoigne le concept de "nation thaïlandaise ", qui est rendu par la combinaison chat sasana phra mahakasat (peuple [au sens de groupe de naissance]-religion [au sens de religion bouddhique] - roi [au sens de chef militaire]). 
Si la royauté est indissociable du concept moderne de nation, c'est certes que les Thaïs ont essentialisé cette institution qui résume à elle seule l'origine, l'histoire et la grandeur du pays, mais c'est aussi parce que la dynastie Chakri, établie depuis 1782, a joué un rôle essentiel dans la construction de la nation thaïlandaise et qu'elle est aujourd'hui encore perçue comme la garante de son unité et de sa stabilité malmenées par de fréquentes crises politiques. Dès le règne de Rama I (1782-1809) les Chakri cherchèrent à moderniser les lois et l'administration du pays. Cependant, leurs initiatives en ce sens prirent un tour décisif sous les règnes de Rama IV (1851-1868) et surtout de Rama V (1868-1910). Ce dernier réussit à contenir la pression des impérialismes français et britannique. Il lança aussi de grands projets d'infrastructures et réforma l'administration et la fiscalité selon le modèle européen. Ce train de réformes toucha aussi la structure sociale avec l'abolition du système formel de patronage sakdina, tandis que l'étiquette de cour était assouplie. Néanmoins ces aménagements ne touchaient en rien l'exercice du pouvoir.

Les recherches consacrées aux réformes conduites sous Rama V montrent qu'elles furent conçues et mises en œuvre à partir de 1885 par un groupe de princes de hauts rangs éduqués à l'étranger et suffisamment loyaux pour faire figure «d'hommes du roi »23. Si ces princes étaient les architectes effectifs de la politique du pays, à l'image du prince Damrong, alors ministre de l'Intérieur et à qui l'on doit la refonte de l'administration des provinces, les grandes orientations étaient tranchées par le roi. Celui-ci, de surcroît, s'érigeait en conscience éclairée du peuple et se pensait seul à même de décider ce qui était bon ou mauvais pour ses sujets, ce qui était compatible ou ne l'était pas avec la culture et l'identité thaïe (khwam pen thai, litt. « être Thai »), et enfin ce qu'il était possible de faire en vertu de la perception très aristocratique qu'il avait des capacités de son peuple. Rama $V$ rejeta ainsi les avis d'une frange de la famille royale lui suggérant de faire évoluer le système politique vers un régime constitutionnel, aux prétextes que le Siam manquait de personnalités compétentes pour composer le Parlement, qu'il serait inutile car ne pouvant contrecarrer son autorité et aurait juste

${ }_{23}$ B. J. Terwiel, A History of Modern Thailand, 1767-1942, St Lucia, University of Queensland Press, 1983, p. 255. 
pour effet négatif de «terrifier» la population ${ }^{24}$. De même, son successeur, Rama VI (1910-1925) refusa que l'économie soit enseignée dans le royaume, selon la thèse que l'enseignement de cette matière risquait de fracturer la société en légitimant l'idée qu'il y aurait des strates de riches opposées à des strates de pauvres. À la science économique, il opposait sa propre philosophie d'inspiration bouddhique selon laquelle chacun devait se contenter de ce que son karma lui avait attribué25. Dans l'optique de ces souverains, seul un monarque bouddhiste droit et juste était en mesure d'assurer la prospérité $\mathrm{du}$ royaume. Or, bien que le pays soit passé à un régime de royauté constitutionnelle suite au coup d'État de 1932, cette interprétation de la fonction royale est devenue partie intégrante de l'idéologie nationale. Elle s'est d'ailleurs ancrée à ce point dans l'imaginaire populaire qu'à partir des années 1990, dans le sillage du grand boom économique du royaume, la statue équestre du roi Chulalongkorn située à Dusit, devant l'ancien Parlement, est devenue un lieu de culte parmi les plus populaires de Bangkok. On s'y rend pour vénérer le "grand modernisateur du pays », initiateur du capitalisme national, et solliciter son soutien occulte dans la conduite des affaires ${ }^{26}$.

Le coup d'État de 1932 n'eut pas pour effet l'instauration d'un réel régime parlementaire, mais prit rapidement la tournure d'une passation de pouvoir du roi aux militaires, d'une monarchie reconnue comme telle à une autre, séculière, affectant les traits de la dictature. Si le maréchal Phibun Songkhram aux affaires de 1938 à 1944, puis de 1948 à 1957, subordonna la royauté à son propre culte, son successeur, Sarit Thannarat (1957-1963) initia une politique de réhabilitation au nom du retour aux valeurs traditionnelles de hiérarchie et d'autorité que les autres militaires aux commandes de l'État poursuivirent jusqu'à la fin des années 1980 et l'arrivée au pouvoir de civils issus des milieux d'affaire. De la fin des années 1950 à la fin des années 1980, période qui

\footnotetext{
${ }^{24}$ D. Wilson, Politics in Thailand, New York-Ithaca, Cornell University Press, 1962, p. 102.

25 T. Winichakul, Siam Mapped, The Geo-Body of a Nation, Honolulu, University of Hawai'i Press, 1994, p. 4.

${ }_{26}$ P. Jackson, «Royal Spirits, Chinese Gods, and Magic Monks: Thailand's Boom-Time Religions of Prosperit ", Southeast Asia Research 7-3, 1999, p. 245-300; I. Stengs, Worshipping the Great Modernizer, King Chulalongkorn, Patron Saint of the Thai Middle Class, SingapourSeattle, NUS Press-University of Washington Press, 2009.
} 
correspond à la première moitié du règne de l'actuel souverain Bhumibol (Rama IX), un système de soutien mutuel associant les généraux et la royauté prit forme et se développa selon des rapports de co-patronage qui évoluèrent dans le temps à l'avantage de l'institution royale. Les militaires à la tête de l'État se posaient en garants de la pérennité de la royauté et affichaient ostensiblement leur déférence à son endroit. En retour, le souverain légitimait implicitement le régime autoritaire en place dans l'accomplissement de ses charges protocolaires, ainsi que par une posture officielle de non-ingérence dans la gestion des affaires courantes, sauf lorsque le recours à son patronage suprême était requis pour dénouer au nom de l'unité nationale et de la paix civile des situations de conflit autrement inextricables. Bhumibol sut tirer un bon parti de cette répartition des rôles. Le fait de ne pas voir son action jugée au gré des vicissitudes de la vie politique et de l'évolution socioéconomique $\mathrm{du}$ pays, lui permit d'accroître progressivement son charisme par un travail soigné sur l'image. Dans les médias nationaux tenus par l'armée il apparut rapidement comme un roi moderne, amateur de jazz, voire à la pointe de l'innovation technologique (par les machines d'oxygénation des eaux polluées qu'il conçut), mais aussi comme un roi bouddhiste compassionnel, mobilisant tous les moyens en son pouvoir pour le bien-être du peuple, la paix civile et la promotion du patrimoine national. Si Sihanouk a pu être perçu comme un « roi aux neuf vies $\gg 27$, tant sa trajectoire politique fut sinueuse, la ligne de conduite de Bhumibol fut à l'inverse d'une grande constance, avec pour effet l'image d'un souverain serein, investi d'une sagesse dharmique qui le préserve des bas calculs politiciens. L'autorité morale qu'il a ainsi acquise, alliée à l'exceptionnelle longévité de son règne (il a été couronné en 1950) l'ont consacré comme un souverain doté d'un karma exceptionnel et comme un grand cakravartin, pivot stable des institutions politiques et capable de réguler l'ordre sociocosmique.

L'image que Bhumibol s'est forgée au fil des décennies est indissociable de ses œuvres sociales ou patrimoniales, et des ressources fournies à cet effet par le Bureau de la Propriété de la Couronne (BPC). Fondé en 1936, le BPC gère les avoirs et les biens fonciers et immobiliers de la Couronne. Des investissements à long terme dans le secteur

${ }^{27}$ R. Kershaw, op. cit., p. 89-100. 
bancaire (Siam Commercial Bank) et dans l'industrie (Siam Cement) ont apporté une manne financière considérable au BPC à partir du décollage économique du pays dans les années 1970-1980. Le Bureau est aujourd'hui le deuxième plus important conglomérat national d'entreprises et dégage de très substantielles marges bénéficiaires chaque année, bien qu'il ait été durement affecté par la crise financière de 1997. Une partie de ses revenus sert à financer les projets de la famille royale. Ceux-ci sont orientés dans plusieurs directions: l'intégration socio-économique et culturelle des minorités montagnardes du Nord ; la promotion du patrimoine architectural et artisanal de la nation par la mise en place de structures de production et de commercialisation de produits de qualité qui assurent des revenus décents aux producteurs ; l'aide au développement économique des agriculteurs des régions pauvres par la promotion de systèmes intégrés, couplés à des structures de microcrédits gérées au niveau communautaire; la protection du patrimoine naturel du pays (forêt, espèces sauvages menacées); ou encore l'éducation de la jeunesse afin de l'associer au renouvellement du patrimoine artistique et artisanal thaï, mais aussi de lui inculquer la philosophie royale de l'économie d'autosuffisance.

La promotion de cette philosophie dont les concepts clefs sont la sécurisation alimentaire, l'autogestion communautaire et la responsabilisation individuelle vise à substituer au cercle vicieux de la recherche individualiste du profit, de la surexploitation de l'homme par l'homme, de l'endettement et de la paupérisation, le cercle vertueux de l'entraide et de la réduction des inégalités au sein des communautés rurales, afin de renforcer leur capacité de résistance face aux velléités prédatrices $\mathrm{du}$ capitalisme sauvage. Cette doctrine peut paraître paradoxale au regard de l'immense fortune bâtie sur un mode capitalistique dont dispose la Couronne. Certes les entreprises gérées par le BPC font figures de modèles en matière de bonne gouvernance et de traitement respectueux de l'environnement, du personnel et de la clientèle. De plus, Bhumibol a toujours affiché un style de vie austère et a su se montrer à l'écoute des difficultés du petit peuple ${ }^{28}$. Enfin, pour la plupart de ses sujets il est «naturel » qu'un souverain doté d'une grande

${ }_{28}$ M. Pellegi, Lord of Things. The Fashioning of the Siamese Monarchy's Modern Image, Honolulu, University of Hawai'i Press, 2002, p. 166. 
vertu (barami) dispose de la plus grosse fortune du pays selon l'entendement qu'ils en ont, surtout lorsqu'il en consacre une partie à des œuvres sociales ${ }^{29}$. Pour autant la doctrine du roi le place en porte-àfaux idéologique par rapport à certaines élites politiques issues des milieux d'affaire, dont Thaksin Shinawatra est devenu la figure emblématique et qui, elles, conduisent depuis la fin des années 1980 une politique résolument néolibérale, tout en se présentant comme les champions d'un processus de démocratisation à l'occidentale. Au nom de la promotion du parlementarisme et d'une gestion managériale du pays mettant l'accent sur les compétences réelles et le volontarisme, ces libéraux ont rapidement clivé la vie politique nationale en s'opposant au conservatisme paternaliste de 1' 'ammat, le cercle des conseillers du palais dont plusieurs sont d'anciens militaires ayant dirigé ou occupé par le passé de hautes fonctions dans l'appareil d'État. Le roi, âgé de 85 ans (en 2013) et qui est très régulièrement hospitalisé depuis 2009 pour de graves problèmes pulmonaires, voit ainsi sa vieille entente contractée avec les généraux, et qui lui a progressivement permis d'exercer un patronage occulte de la vie politique, publiquement contestée par des politiciens affairistes en contrepoint desquels il a tout au long de son règne bâti sa stature morale. La royauté qui faisait figure de pivot stable des institutions et d'arbitre suprême des conflits devint implicitement matière à débat.

Le clivage prit une tournure plus radicale lorsqu'en 2006 Thaksin, au faîte de sa popularité, contesta ouvertement le pouvoir de 1'ammat. Quelques mois plus tard il fut renversé par un coup d'État militaire et accusa d'être à l'origine de son éviction le président du conseil privé du roi, le général Prem Tinsulanonda, ancien Premier ministre de 1980 à 1988. Ce coup de force exacerba les tensions et aboutit dans les années qui suivirent à des manifestations monstres mobilisant au gré des gouvernements en place, soit les partisans de la royauté (les Chemises jaunes), soit les partisans de Thaksin (les Chemises rouges). Depuis les élections législatives de 2011 et le retour indirect au pouvoir de Thaksin par l'entremise de sa sœur cadette, Yingluck Shinawatra, un modus

${ }^{29}$ Dans les faits, il convient de distinguer la propriété privée du roi, gérée par l'Office de la propriété privée, des biens de la Couronne gérés par le BPC et dont les revenus, hors investissements, sont exclusivement consacrés aux œuvres sociales, culturelles et environnementales de la famille royale. 
vivendi précaire s'est instauré entre les deux pôles antagonistes qui structurent aujourd'hui la vie politique nationale. Dans le sens d'un apaisement des tensions, la constellation hétéroclite de groupes de pression pro-Thaksin, que constitue l'Union Démocratique contre la Dictature (UDD), a pris soin d'inscrire en tête de son programme en six points l'objectif d'une démocratie ayant le roi comme chef de l'État, avec cependant comme bémol que «la souveraineté appartienne véritablement au peuple ${ }^{30}$.

À terme l'avenir de la royauté en Thaïlande est-il en question ? Le roi Bhumibol reste vénéré par la très grande majorité de la population. Peter Jackson ${ }^{31}$ a sans doute raison d'écrire que le culte actuel à Chulalongkorn préfigure celui qui sera voué plus tard à Rama IX, tant celui-ci aura marqué de son empreinte l'histoire contemporaine du pays. Le prince héritier, Maha Vajiralongkorn, s'investit peu dans les œuvres royales et souffre d'un déficit de popularité. On le dit plus conciliant à l'endroit de Thaksin avec en perspective la possibilité d'un pacte d'alliance d'un nouveau genre avec les milieux d'affaire. Quant aux œuvres sociales et patrimoniales de la famille royale qui ont largement contribué à sa popularité, elles sont depuis plusieurs années déjà supervisées par la princesse Sirindhorn qui est la mieux à même de pérenniser le capital symbolique accumulé par son père.

\section{3 - Le sultanat de Brunei}

Brunei est le seul État sud-est asiatique qui se définisse aujourd'hui explicitement par l'exercice du pouvoir royal. L'idéologie d'État, dont les prémisses ont été élaborées dès le début des années 1950 par le sultan Omar Ali Saifuddin III, avant d'être mise en œuvre par l'actuel souverain en 1991, s'intitule sous sa forme anglaise Malay Islamic Monarchy. L'examen de sa formulation vernaculaire, Melayu Islam

\footnotetext{
${ }^{30}$ E. Mérieau, Les Chemises Rouges de Thailande, Bangkok, « Carnets de l'Irasec », n 23, 2013, p. 73 .

${ }^{31}$ P. Jackson, op. cit., p. 298.
} 
Beraja ${ }^{32}$ ou MIB, i.e. «Malais [égal] musulman et pourvu d'un roi » révèle à l'inverse ce qui en jeu n'est pas tant la nature du régime, que la construction d'une identité nationale. Bien que, selon le Coran « détenir la royauté [malik al mulk] » soit le seul privilège de Dieu (Surat III, 25), le titre de roi s'est répandu dans certains des émirats subordonnés au califat abbasside sous l'influence persane, en parallèle avec celui de sultan (" celui qui commande) », utilisé depuis le XI" siècle 33 , qui devient dans l'Inde musulmane l'équivalent de raja. . Si au Brunei le souverain porte ainsi le titre de sultan au côté de son équivalent malais de Yang Dipertuan ("celui qui est le maître»), l'essentiel des titulatures, la symbolique et les rituels royaux demeurent pourtant décalqués de modèles indianisés.

Les quelques rares sources textuelles et archéologiques disponibles $^{34}$ montrent en effet que la chefferie installée sur la rivière de Brunei fut très progressivement indianisée à partir de la seconde moitié du premier millénaire de notre ère, avant d'adopter l'islam au tout début du XVe siècle.

Le sultanat, qui se réclamait doublement du modèle acihais et d'une alliance fondatrice avec Johor (c'est-à-dire avec Malaka), était administré au moins depuis le XVIIe siècle, par quatre grands dignitaires issus de la famille royale, Bendahara, Temenggong, Di Gadong et Pemancha. Le Bendahara et le Temenggong coiffaient chacun un premier niveau de huit cheteria ${ }^{35}$ princiers et un deuxième de huit menteri ${ }^{36}$, ce qui faisait un total de seize cheteria et de seize menteri, répartis par moitié entre les deux dignitaires. On retrouve ainsi à Brunei un dispositif par cercles concentriques de type mandalaïque, caractéristique des royaumes indianisés.

32 Melayu Islam Beraja, Jiwa Raga Brunei [Le MIB, corps et âme du Brunei], Bandar Seri Begawan, Jawatankuasa Penyiaran, Penerangan dan Percetakan Majlis Ilmu [Publication et édition du Conseil Scientifique], 2010, 497 p.

33 Voir Janine et Dominique Sourdel, Dictionnaire historique de l'Islam, Paris, PuF, 1996, p. 179-198, 523-4,774-5.

${ }^{34}$ M.-S. de Vienne, Brunei, de la thalassocratie à la rente, Paris, CNRS Éditions-Irasec, 2012, p. 21-23.

${ }^{35}$ Dignité nobiliaire.

${ }^{36} \mathrm{Du}$ sanscrit mantri (celui qui maîtrise les mantra), menteri est une dignité honorifique conférée aux roturiers assumant des responsabilités administratives. 
La déliquescence du sultanat, puis la résidence britannique (19061959) ayant dissocié les fondements de la gestion des hommes de ceux de la Royauté, les souverains se sont efforcés dès qu'ils en ont eu les moyens de restaurer un lien direct avec la population en reconstituant une tradition royale tombée en décrépitude. Ils ont "amélioré » les anciens rituels ${ }^{37}$ en collationnant la documentation relative au coutumier palatin pour convoquer des pratiques remontant à Malaka, puisées dans les sources malaises comme dans les travaux occidentaux.

Est ainsi formalisée sous le sultan Ahmad Tajuddin (r. 1924-1950) une dyarchie cérémonielle - statutairement asymétrique - réunissant par moitié les «nobles», qui détiennent le pouvoir de décision, et leurs inférieurs, les menteri "roturiers", qui gèrent le territoire. Le système trouve sa cohérence dans l'articulation des deux hiérarchies parallèles des nobles et des roturiers par l'intermédiaire de quatre menteri: les deux chefs-menteri du Di Gadong, et les chefs-menteri du Bendahara et du Temenggong. Le jeu hiérarchique reconstitue ainsi le lien social entre les deux moitiés de la société, les roturiers et les nobles, mis à mal par la surexploitation des populations riveraines par les seconds au XIXe siècle, tout en incorporant, au moins symboliquement, les tenants d'une administration «moderne» dans la hiérarchie traditionnelle. Inconnu des cours malaises de Péninsule, ce dyarchisme s'inspire à l'évidence des sociétés « à hiérarchie » de l'ouest et du centre de Bornéo, singulièrement des Kenyah où l'on retrouve une stratification à cinq degrés, inscrite spatialement dans la longue maison ${ }^{38}$. De fait, le terme brunéien utilisé pour désigner les nobles de second rang est cheteria damit (" petit aristocrate ») quand les Kenyah appellent leur deuxième rang $\operatorname{deta}^{\prime} u$ dumit ("petit chef»); quant aux roturiers, ils sont désignés chez les Kenyah (et Kayan) par le terme de panyin, ce qui renvoie à la plus haute dignité à laquelle ils peuvent accéder au Brunei, celle de pehin ${ }^{39}$.

\footnotetext{
37 Jamil al-Sufri, Pehin Dr. Hj. Mohd., Adat istiadat diraja Brunei, BSB, Jabatan Adat Istiadat Negara, 2002, " exergue », page de garde.

38 Bernard Sellato, "Note préliminaire sur les sociétés "à maison" à Bornéo ", in C. Macdonald (dir.), De la Hutte au Palais, Sociétés 'à maison' en Asie du Sud-Est insulaire, Paris, Presses du CNRS, 1987, p. 15-44.

39 Titre conféré aux roturiers pour services rendus, soit aux 64 menteri plus à divers dignitaires ethniques dont les deux responsables de la communauté chinoise.
} 
Omar Ali Saifuddin engage ensuite dès 1954 un redéploiement de la symbolique royale avec l'instauration d'un département de la coutume (adat istiadat). L'interface entre la noblesse de second ordre et les plus élevés en rang des roturiers devient ainsi le point articulatoire de la mobilité sociale. En 1958, ce système est parachevé par la création de quatre grades multiples de quatre (4 dignitaires du premier rang; $8 \mathrm{du}$ second ; 16 du troisième et 32 du quatrième) chez les cheteria et menteri, quand l'articulation formelle entre les deux moitiés, noble et roturière, du dispositif, disparaît. La notitia dignitatum perd ainsi ses derniers éléments fonctionnels au profit d'un renforcement de sa charge symbolique : placer le souverain au cœur d'un dispositif cosmologique à quatre degrés et à quatre auréoles dont il constitue à la fois le centre et le pivot, et dont le trône constitue l'une des représentations. Ce symbolisme est renforcé par l'application d'un schéma de même ordre aux dignitaires religieux, placés sous supervision directe du souverain depuis 1994.

La fabrique des rangs se poursuit jusqu'à ce jour sous le règne du sultan Hassanal Bolkiah (1967-). Comme ces derniers sont loin d'être tous conférés, c'est bien l'existence même du dispositif hiérarchique qui est pertinent: en distinguant les honneurs de la Cour des charges administratives, il prévient l'attribution de postes à des personnes dont la «qualité» ne garantit pas toujours le savoir-faire, tout en reconnaissant aux détenteurs de compétences une place, fut-elle modeste, dans la grille statutaire.

De multiples célébrations, dont les modalités sont à l'inverse très largement novatrices, mettent régulièrement le souverain en contact avec ses sujets : lors de Hari Raya - qui célèbre la fin du jeûne et le début du mois de Shawal - le quart de la population du sultanat (y compris des enfants et des immigrés) défile pendant trois jours pour saluer les souverains. Les diplômes universitaires sont remis personnellement par le Sultan, lequel assiste chaque semaine au prêche du vendredi dans une mosquée différente. Il est présent à chaque inauguration d'usine, de bâtiment administratif ou social d'importance. La multiplication des célébrations permet également de promouvoir un fonctionnement moderne par les corps, en faisant parader lors de fêtes religieuses comme le Maulud (la naissance du Prophète) ou civile (fête nationale) des représentants de toutes les composantes de la société. 
Portée par la rente pétro-gazière, l'invention de la tradition royale s'est ainsi retrouvée au cœur du processus de modernisation socioéconomique d'un État où le sultan est à la fois Premier ministre, ministre des Finances, ministre de la Défense et chancelier de l'Universiti Brunei Darussalam, le Conseil législatif, pour l'essentiel nommé, siégeant une douzaine de jours par an.

\section{Conclusion : vers une histoire longue des systèmes politiques}

Il ne s'agit plus, comme le fit Robert Heine-Geldern en 1942, d'énoncer comment les royautés procèderaient d'un modèle univoque. En raison de leur commun héritage indianisé, restructuré d'un côté par le bouddhisme theravāda, et de l'autre par l'islam, elles arborent assurément des formes rituelles et symboliques similaires (cosmologies quinaires, abhisheka $a^{40}$ royales, présence d'un corps sacerdotal sacrifiant aux cultes royaux dérivé du principe des purohita ou "chapelain royaux», etc.). Il n'en reste pas moins qu'elles sont le produit de trajectoires irréductibles les unes aux autres, dont seule une histoire longue permettrait de rendre compte. Considérer les systèmes politiques contemporains à la lumière de leur héritage moderne (XVIe-XIXe siècles) est à cet égard essentiel.

La vieille royauté cambodgienne offre l'exemple d'un premier paradoxe : institutionnellement la plus faible, elle a survécu en raison du jeu mécanique d'un factionnalisme endémique qui n'a cessé d'être la colonne vertébrale de l'histoire contemporaine, selon une dialectique mise en place sous le règne de Ang Eng (r. 1772-1797). Malgré la culture d'extrême violence politique qui préside aux destinées du pays depuis la fin du XVIII siècle, le Cambodge a ainsi pu préserver certaines modalités du lien social, quand bien même le "développement" économique amorcé depuis la signature des Accords de Paris (1991) opère aujourd'hui des ravages. La jeune royauté thaïlandaise donne à voir un paradoxe inversé : bâtie sur un roc institutionnel puisant à

\footnotetext{
${ }^{40}$ abhișeka (ondoiement du roi).
} 
diverses traditions régionales, elle vacille aujourd'hui devant les contradictions d'un fonctionnement par crises politiques cycliques ${ }^{41}$, habituellement régulé par une ostentation de la figure royale signifiant la primauté de l'ordre sur le chaos. Que ce vacillement intervienne en période de succession n'est cependant pas pour étonner: elle est toujours critique pour les royautés dépourvues de règle explicite de dévolution. Entre le bambou khmer qui ne rompt pas sous la charge et le ficus thaïlandais menacé d'être emporté par les vents, le cas de Brunei tranche par sa stabilité. Il est, certes, difficile de jauger une petite entité de la taille de la Corse contrôlant quelques centaines de milliers de personnes à l'aune d'appareils gérant des densités humaines autrement plus importantes. La reconstruction d'un appareil cérémoniel reliant le sommet de l'appareil d'État aux strates les plus pauvres de la population de citoyenneté brunéienne est sans nul doute une dimension de l'équation; une autre en est le cocon social protecteur édifié par la « royauté-providence ».

${ }^{41}$ M.-S. de Vienne, «Thaïlande, pour une relecture du jeu politique », Péninsule n 24-25, 1992 (1-2), p. 171-213 ; Jean Baffie, « Une "démocratie" entre populisme et défiance envers le peuple: la politique en Thaillande depuis la Seconde Guerre mondiale », in Stéphane Dovert et Jacques Ivanoff (dir.), Thailande contemporaine, Paris-Bangkok, Les Indes savantesIrasec, 2011, p. 139-200. 


\section{Pour en savoir plus}

\section{1 - Les modèles politiques}

MOUSNIER, Roland, Monarchies et royautés de la préhistoire à nos jours, Paris, Librairie Académique Perrin, « Pour l'Histoire », 1989.

DuMONT, Louis, "Appendice C: La conception de la royauté dans l'Inde ancienne ", in Homo hierarchicus. Le système des castes et ses implications, Paris, Gallimard, Tell, 1966, p. 351-375.

TAMBIAH, Stanley Jeyaraja, «The Galactic Polity: The Structure of Traditional Kingdoms in Southeast Asia ", Annals of the New-York Academy of Sciences, vol. 293, 1977, p. 69-87.

\section{2 - L'incarnation historique des modèles}

LOMBARD, Denys, "Le sultanat malais comme modèle socio-économique », [in] Denys Lombard et Jean Aubin (dir.), Marchands et hommes d'affaires asiatiques dans l'océan indien et la Mer de Chine, XIIIe-XXe siècles, Paris, EHESS, 1988, p. 117-124.

FOREST Alain, « Le processus traditionnel de légitimation du pouvoir royal dans les pays de Bouddhisme Theravada (Thaïlande, Cambodge) », Journal des Anthropologues. Anthropologie et histoire face aux légitimations politiques, $\mathrm{n}^{\circ} 104-$ 105, 2006, p. 165-189.

\section{3 - Exemplum sud-est asiatique}

BRown, D. E., Brunei : the structure and history of a Bornean Malay Sultanate, Brunei, Brunei Museum, 1970, $239 \mathrm{p}$.

NÉPOTE, Jacques, État présent de la maison royale du Cambodge, Paris, Institut de la Maison Royale du Cambodge, 1994, 154 p.

VICKERY, Michael, "The Future of Thailand?», 1977, édition révisée avec commentaires additionnels, publication numérique inédite, 2011, http:/ / michaelvickery.org/vickery1977future.pdf 